\title{
Insensitivity of trapping at surfaces to molecular vibration
}

\author{
Alec M. Wodtke ${ }^{\mathrm{a}, *}$, Huang Yuhui ${ }^{\mathrm{b}}$, Daniel J. Auerbach ${ }^{\mathrm{c}}$ \\ ${ }^{a}$ Department of Chemistry and Biochemistry, University of California at Santa Barbara, 4623C Physical Sciences, \\ North Santa Barbara, CA 93106, United States \\ b Oplink Communications, 46335 Landing Parkway, Fremont, CA 94538-6407, United States \\ ${ }^{\mathrm{c}}$ Hitachi Global Storage Technologies, 650 Harry Road, San Jose, CA 95120-6099, United States
}

Received 14 May 2005; in final form 6 June 2005

Available online 19 August 2005

\begin{abstract}
We have measured the trapping probability of $\mathrm{NO}(v=0$ and $v=2)$ on $\mathrm{Au}\left(\begin{array}{lll}1 & 1 & 1\end{array}\right)$ as a function of translational energy of incidence. The range of translational and vibrational energies probed is comparable, yet the influence of the two types of motion is very different; $0.46 \mathrm{eV}$ of translational energy is sufficient to completely suppress trapping, but the $0.46 \mathrm{eV}$ of vibrational energy in $\mathrm{NO}(v=2)$ has no measurable effect. The insensitivity of trapping probabilities to vibrational energy stands in sharp contrast to the strong sensitivity of direct activated dissociative adsorption to vibrational energy.
\end{abstract}

(C) 2005 Elsevier B.V. All rights reserved.

Trapping, the process whereby an atom or molecule incident on a surface loses sufficient kinetic energy to become bound in a weakly adsorbed state, is an important and widely studied elementary step in surface physics and chemistry [1-3]. A detailed understanding of trapping is important for several reasons not the least of which is its relevance to surface chemistry and heterogeneous catalysis. Chemisorption and molecular dissociation at surfaces often involve a sequential process where incident molecules first trap into a weekly bound and mobile precursor state [4-8], then move on the surface to a favorable site or configuration, and finally chemisorb or dissociate.

In addition to its role in dissociative adsorption, trapping is also interesting in its own right as a probe of molecular interactions and energy transfer dynamics at surfaces $[1,9,10]$. Indeed, the translational energy dependence of trapping probabilities is a very sensitive measure of the strength of molecule-surface interactions and the efficiency of energy transfer during molecule-

\footnotetext{
* Corresponding author. Fax: +1 8058934120.

E-mail address: wodtke@chem.ucsb.edu (A.M. Wodtke).
}

surface collisions. A related basic issue is the question of the applicability of statistical theories of reaction rates to trapping. While it is tempting to invoke the large number of atoms in a solid surface (and the associated large density of vibrational states) as an overriding factor suggesting trapping on a metal surface will behave statistically, the situation is more complicated. Only a limited number of surface atoms interact with the incident molecule and surface phonons are known to couple only weakly to adsorbate vibration [11-13]. In the absence of good experimental observations, this question of the applicability of statistical theories remains open.

The importance of understanding trapping has motivated a great deal of experimental and theoretical work on the subject. Indeed, there is an extensive literature on trapping probabilities and their dependence on the angle and kinetic energy of incident atoms and molecules [1-3,14]. In contrast, the question of how trapping depends on the vibrational state of an incident molecule has received almost no attention. In fact, we are not aware of any experiments that directly probe the vibrational state dependence of trapping probabilities. This 
lack of information about the effect of molecular vibration on trapping is unfortunate, since a complete understanding of interactions at surfaces must include knowledge of the role of molecular vibration. Indeed, the vibrational state of incident molecules often plays a role of comparable importance as their kinetic energy in direct dissociative adsorption at surfaces [15-18]. Furthermore, careful analysis of experimental trapping probabilities and their dependence on vibrational and translational incidence energy represents an attractive benchmark to test theoretical methods that hope to simulate surface adsorption. For example, theoretical simulations of $\mathrm{NO}$ on $\mathrm{Ag}\left(\begin{array}{lll}1 & 1 & 1\end{array}\right)$ using empirical potential energy surfaces predicted vibration has little influence on trapping [19]. Experiments capable of measuring such influences would be quite helpful in evaluating the validity of such simulations.

Here, we present the first measurements of the influence of vibrational energy on trapping. We measure and compare the effect of vibrational and translational energy of incidence on trapping of NO molecules at an $\mathrm{Au}\left(\begin{array}{lll}1 & 1 & 1\end{array}\right)$ metal surface. The results show a strong dependence of trapping on translational energy, as expected, but a remarkable insensitivity to the vibrational state of the incident molecules. This insensitivity to vibration stands in stark contrast to the strong influence of vibration on activated (non-precursor mediated) dissociative adsorption, where many experiments have shown that vibration has a comparable or even larger influence to translation.

The experiment involves measuring the flux and quantum state distribution of scattered NO molecules initially in the $v=0$ and $v=2$ vibrational state for various incidence energies. The surface temperature $(300 \mathrm{~K})$ and the binding energy $(0.5-0.8 \mathrm{eV})$, which have recently been calculated by S. Roy and J.C. Tully and privately communicated to us, mean that we operate in a regime where the residence times on the surface are longer than $30 \mu$ s. Thus, if vibrationally excited molecules trap to the surface, they will desorb after having reached thermal equilibrium with the surface, i.e. in $v=0$. Therefore, by monitoring in separate experiments: (1) the reflection probability of $v=2$ and (2) the fraction of molecules that are transferred from $v=2$ to $v=1$, we are able to extract the trapping probability for $v=2$. In this analysis, it is assumed that direct scattering channel (i.e. without trapping and desorption) transferring population from $v=2$ to $v=0$ is not important. This was previously shown to be correct at incidence energies of translation above $\sim 35 \mathrm{~kJ} / \mathrm{mol}$ [20]. As we expect, the role of direct vibrationally inelastic channels to become less important as translational energy of incidence is reduced [21], this assumption would appear to be valid at those energies below $35 \mathrm{~kJ} / \mathrm{mol}$ as well. The trapping probability for $v=0$ is obtained again by monitoring the reflection probability of $v=0$. These experiments are then carried out at several incidence energies of translation. With this approach, we are able to unambiguously extract the trapping probability as a function of incidence energy for individual vibrational states. The design of the experiments probes a range of vibrational energy similar to the range of incident energies, facilitating a useful comparison.

We have previously reported evidence of trapping of $\mathrm{NO}$ on $\mathrm{Au}\left(\begin{array}{lll}1 & 1 & 1\end{array}\right)$ [20], but the subject of that paper was the role of electron hole pairs in vibrational energy transfer. Here, we take up the matter of vibrational and translational influences on trapping. The experimental arrangement has been described in detail [20,22-24]. Briefly, $\operatorname{NO}(v=2, J=1.5, \Omega=0.5)$ in both elf parity states was prepared by infrared overtone pumping of a pulsed supersonic beam of NO $28-\mathrm{cm}$ upstream from a $\mathrm{Au}\left(\begin{array}{lll}1 & 1 & 1\end{array}\right)$ single crystal surface. For the IR pumping needed in this work, we generated $2.7 \mu \mathrm{m}$ $\left(3729 \mathrm{~cm}^{-1}\right)$ light $\left(0.08 \mathrm{~cm}^{-1}\right.$ line-width, $\sim 6 \mathrm{~mJ} /$ pulse, 5-ns pulse duration) by mixing in a $\mathrm{LiNbO}_{3}$ crystal: $120 \mathrm{~mJ} /$ pulse of 761.8-nm light from a Nd:YAG pumped dye laser (operating on LDS-765) and a comparable quantity of the injection seeded $1.064-\mu \mathrm{m}$ light produced by the fundamental of the Nd:YAG laser. We estimate $\sim 2 \%$ of the total population in the illuminated part of the molecular beam is pumped to the desired state. The supersonic NO molecular beam was generated using a pulsed molecular beam source [25], collimated by passage through three differentially pumped stages and impinged at normal incidence upon a $\mathrm{Au}\left(\begin{array}{lll}1 & 1 & 1\end{array}\right)$ crystal mounted in an ultra-high-vacuum. Seeding the $\mathrm{NO}$ in $\mathrm{He}, \mathrm{Ar}, \mathrm{Kr}$, and $\mathrm{H}_{2}$ allowed us to vary the kinetic energy of the NO. The Au(lllll 111$)$ surface was cleaned daily by sputtering with $2.8 \mathrm{kV} \mathrm{Ar}{ }^{+}$for $30 \mathrm{~min}$ and flash annealing to $1100 \mathrm{~K}$. After 9-h exposure to the NO beam, surface contamination was still below the detection limit of Auger electron spectroscopy (AES). Even so, we annealed the surface between each experimental run (approximate every $2 \mathrm{~h}$ ).

We detected either the laser-prepared state of $\mathrm{NO}$ or states resulting from scattering at the surface with one-color, two-photon REMPI through the $\mathrm{A}^{2} \Sigma^{+} \leftarrow$ $\mathrm{X}^{2} \Pi_{i} \gamma$-bands [26,27] using a probe laser positioned $1-2 \mathrm{~cm}$ from the surface. When NO strikes the surface, it can either scatter directly or trap and later desorb. Estimates of the residence time ( $30 \mu \mathrm{s}$ to $300 \mathrm{~ms})$ can be made using the formula, $10^{13} \mathrm{e}^{-\left(E_{\mathrm{a}} / k T\right)}$ and recently calculated binding energies of $0.5-0.8 \mathrm{eV}$ (see above). The directly scattered molecules undergo rotational excitation and may also undergo vibrationally inelastic scattering. By summing over the rotational state distribution of the scattered molecules, we can determine the fraction that survive in the initial vibrational state as well as the fraction that undergo direct vibrationally inelastic scattering from $v=2$ to $v=1$. The trapping probability is then given by 
$P_{\mathrm{v}}^{\text {trap }}=1-P_{\mathrm{v}}^{\text {survival }}-P_{\mathrm{v}}^{\text {excitation }}-P_{\mathrm{v}}^{\text {loss }}$,

where $P_{\mathrm{v}}^{\text {trap }}$ is the trapping probability, $P_{\mathrm{v}}^{\text {excitation }}$ is the probability of vibrational excitation, and $P_{\mathrm{v}}^{\text {loss }}$ is the probability of vibrational de-excitation for molecules in the initial vibrational state $\mathrm{v}$.

The probabilities that laser prepared $\mathrm{NO}(v=2)$ and $\mathrm{NO}(v=0)$ survive collisions with the surface and remain in the same vibrational state are shown in Fig. 1. Here, the A-X $(0,2)$ REMPI signal intensities of the $R_{11}(1.5)$ transition of the incoming beam and several wellresolved transitions of the scattered products were measured and calibrated for the laser powers used. The influence of: angular and TOF distributions, MCP gain differences and rotational partition functions were all taken into consideration. The measured survival probabilities for $\mathrm{NO}(v=0)(--)$ and $\mathrm{NO}(v=2)(--\boldsymbol{\Delta}--)$ were found to be well fit (solid and dashed lines) by the empirical functions

$P_{\text {survival, } v=0}\left[E_{\mathrm{i}}\right]=1.002\left(\mathrm{e}^{-0.0164 E_{\mathrm{i}}}-\mathrm{e}^{-9.586 E_{\mathrm{i}}}\right)$,

$P_{\text {survival, } v=2}\left[E_{\mathrm{i}}\right]=1.114\left(\mathrm{e}^{-0.825 E_{\mathrm{i}}}-\mathrm{e}^{-8.085 E_{\mathrm{i}}}\right)$,

where $E_{\mathrm{i}}$ is the translational energy of the incident $\mathrm{NO}$ in $\mathrm{eV}$. Visual inspection of the $v=0$ results $(--)$ show that complete survival obtains at high incidence energies with a characteristic turn-over energy of $\sim 0.1 \mathrm{eV}$. This reflects the fact that vibrational excitation of $\mathrm{NO}(v=0)$ is negligible even at the highest incidence energies [20]. In the limiting case where vibrational relaxation and excitation of $\mathrm{NO}(v=2)$ were negligible and in the limit of no vibrational influence on trapping, one would expect the $v=2$ results (- $-\mathbf{\Delta}--)$ to overlay those of $v=0$. Casual inspection of Fig. 1 shows this not to be the case. To understand better the meaning

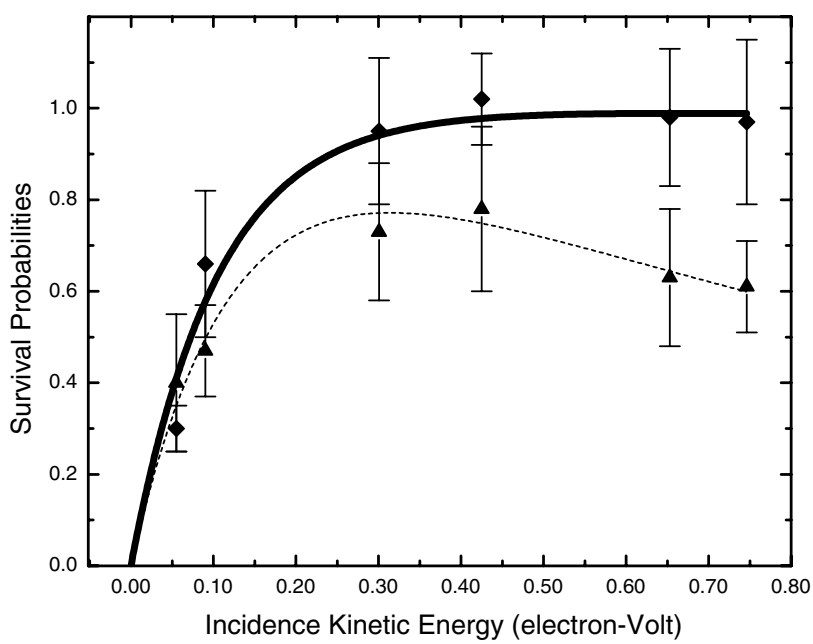

Fig. 1. Vibrational survival probabilities as a function of translational energy for $\operatorname{NO}(v=0)$ and $\operatorname{NO}(v=2)$ incident on $\operatorname{Au}\left(\begin{array}{lll}1 & 1 & 1\end{array}\right)$. Experimentally observed survival probabilities for $\mathrm{NO}(v=0, \bullet)$ and $\mathrm{NO}$ $(v=2, \mathbf{\Delta})$, with error bars. Solid lines indicate empirical fits with the proper asymptotic form represented by Eqs. (2a) and (2b). See text. of this difference, we must take into account the vibrationally inelastic (direct scattering of $v=2$ to $v=1$ ) contributions to the data.

The probabilities of vibrational energy excitation and loss were also reported previously [20]. For the conditions considered here, the probability of vibrational excitation is always less then $2 \times 10^{-4}$ and thus may be neglected. Thus, we must only characterize the term $1-P_{\mathrm{v}}^{\text {loss }}$ in Eq. (1) in order to extract the trapping probability. In Fig. 2, we show this term, which is closely related to the vibrational branching ratio for $\mathrm{NO}(v=2)$ and $\mathrm{NO}(v=1)$ determined in the previous measurements [20]. These measurements were carried out at two surface temperatures, but owing to the lack of surface temperature dependence for vibrational relaxation, both sets of data may be used for the present analysis. To this end, we fitted the data to a straight-line (also shown in Fig. 2).

Using Eqs. (1) and (2b), we may determine by difference the incidence energy dependence of the trapping for $\mathrm{NO}(v=2), P_{\mathrm{v}}^{\text {trap }}(v=2)$. The trapping probability for $\mathrm{NO}(v=0)$ is found more easily by taking the difference of unity and the function in Eq. (2a). The results are shown in Fig. 3, $(\mathrm{NO}(v=0)-$ solid line; $\mathrm{NO}(v=2)$ - dashed line).

As expected, the trapping probability depends strongly on the translational energy of the incident NO decreasing to a $1 / e$ value at $E_{\mathrm{i}} \sim 0.1 \mathrm{eV}$. The dependence of the trapping probability on vibrational energy is much weaker. Indeed, within the limitations of this experiment, there is no observable vibrational influence. In passing we note that this agreement confirms the earlier assumption that direct scattering from $v=2$ to $v=0$ is unimportant below $35 \mathrm{~kJ} / \mathrm{mol}$ incidence energy of translation.

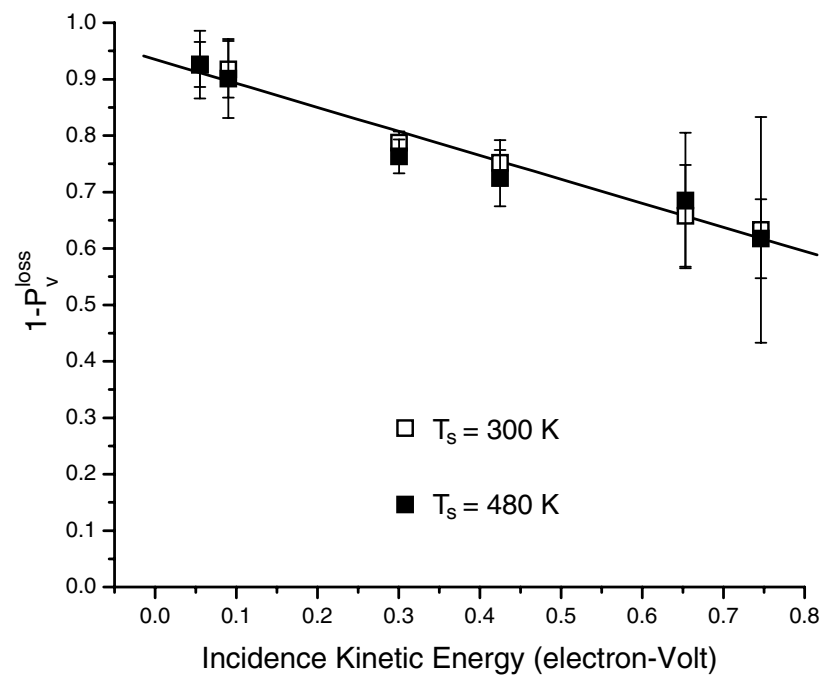

Fig. 2. The term $1-P_{\mathrm{v}}^{\text {loss }}$ (fraction not trapped or lost to vibrational relaxation) from Eq. (1). vs. translational energy for $\operatorname{NO}(v=2)$ incident on $\mathrm{Au}\left(\begin{array}{llll}1 & 1 & 1\end{array}\right)$. Results at two surface temperatures ( $T_{\mathrm{s}}=300 \mathrm{~K}, \square$ and $T_{\mathrm{s}}=480 \mathrm{~K}, \square$ ). The solid line is a least squares fit to both sets of data. 


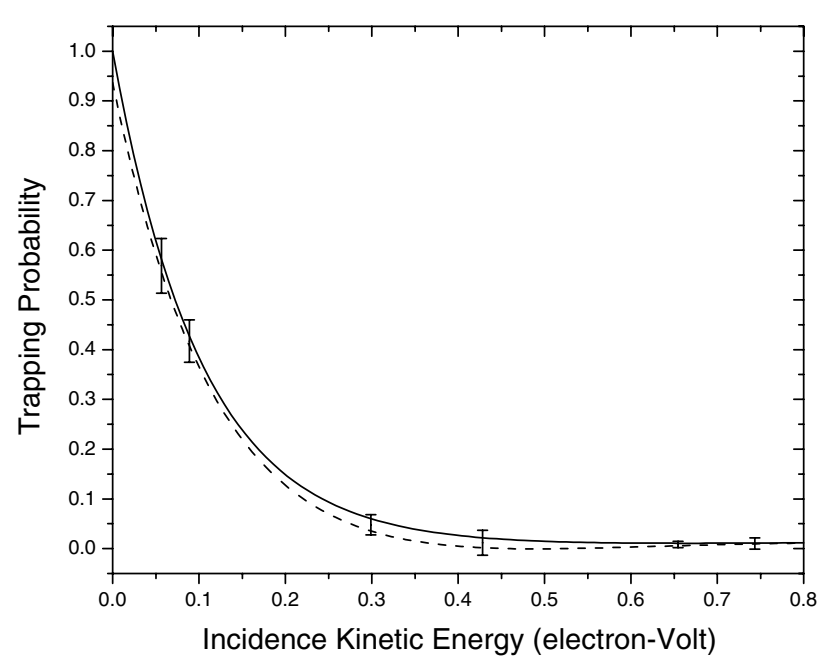

Fig. 3. Trapping probabilities as a function of translational energy for $\mathrm{NO}(v=0)$ and $\mathrm{NO}(v=2)$ incident on $\operatorname{Au}\left(\begin{array}{lll}1 & 1 & 1\end{array}\right)$. The deduced incidence energy dependence for trapping is shown for of $\mathrm{NO}(v=0$, solid line) and $\operatorname{NO}(v=2$, dashed line). Here, one can see that the vibrational energy of $\mathrm{NO}(v=2)$, which is $0.46 \mathrm{eV}$, has no detectable influence on trapping, whereas a similar degree of translational excitation suppresses trapping below the detection limit of the experiment.

$\mathrm{NO}(v=2)$ contains $0.46 \mathrm{eV}$ of excitation energy, an amount that does not suppress trapping to a measurable degree; in contrast, $0.46 \mathrm{eV}$ of translation energy is enough to suppress trapping to below the detection limits of the experiment. These results are also consistent with the conclusion that when $\operatorname{NO}(v=2)$ is trapped it does not emerge as $\operatorname{NO}(v=1)$, indicating that trapping is the only mechanism for multiquantum vibrational relaxation to the ground vibrational state under these conditions.

We are not aware of any previous direct measurements of the vibrational state dependent trapping probability. There are, however, previous experimental results that provide indirect qualitative support for our finding that vibrational energy does not have a strong effect on trapping. Trapping would be influenced if and only if there was a strong 'mechanical' coupling of NO vibration to translational motion. There is some evidence that this coupling may be quite small for NO. First, vibrational excitation probabilities are small for NO collisions with cold metal surfaces, even at translational incidence energies of five times the vibrational energy of $\mathrm{NO}(v=1)$ [28]. More direct evidence can in principle be obtained by measuring $\mathrm{NO}$ velocity changes associated with vibrationally inelastic surface collisions. There have been two attempts to observe such velocity changes for NO in collisions at metals, $\operatorname{Ag}\left(\begin{array}{lll}1 & 1 & 1\end{array}\right)$ [29] and $\mathrm{Au}\left(\begin{array}{lll}1 & 1 & 1\end{array}\right)$ [22]. Neither of these studies showed strong energy exchange between vibration and translation. Unfortunately in both of these experiments, it is difficult to draw firm conclusions due to the limited translational energy resolution and the complications that arise from the strong coupling of translational motion to phonon and rotational excitation. The observations made in the present work are much more sensitive to the role of vibration-translation coupling in gas-surface collisions and place a much stricter limit on the insensitivity of trapping to vibrational excitation than do previous experiments.

For other molecules, the role of vibration on trapping need not be similar to the present case. See for example the case of $\mathrm{NH}_{3}$ umbrella-vibration in collisions with $\mathrm{Au}\left(\begin{array}{lll}1 & 1 & 1\end{array}\right)$ [30] or some of the many studies that report a strong coupling of translational motion to vibrational excitation [14,31] for molecules with low lying vibrational states such as alkanes. For these systems, we might expect a significant effect of vibrational state on trapping probabilities. For these kinds of systems with lower frequency vibrations, it is also worth noting that typical temperatures for reactions at surfaces would mean reacting systems that contain a wide variety of vibrational quantum states. Clearly, it would be interesting to perform experiments on the vibrational dependence of trapping for these kinds of systems.

Discovery of systems where vibrational influences on trapping are large leads to striking predictions. For example, if trapping depended on vibrational state, thermal desorption would exhibit non-Boltzmann vibrational state distributions. Reports of non-Boltzmann vibrational state distributions for $\mathrm{NO}$ can be found $[32,33]$. It is not, however, clear whether all molecules detected in these experiments were trapped and then desorbed or if there is a mixture of direct and indirect scattering.

It is also interesting to compare our results to theoretical studies. DFT calculations that are commonly used to characterize the interaction potentials of molecules with metal surfaces suffer ubiquitous 'over-binding'. Progress will be accelerated by comparisons to data such as that shown here, where first principals trapping simulations can be rigorously tested.

A central question in developing an atomic-scale view of chemistry at solid interfaces concerns the dynamics of trapping. Specifically, what are the microscopic forces that determine whether a molecule is adsorbed or reflected and how do specific kinds of molecular motions influence sticking? Here, we have considered the influence of vibrational vs. translational energy of incidence on trapping of a diatom at a metal surface. We approach this problem using selective laser state preparation and detection methods, in combination with molecular beams that allow us to clearly see the influence of vibration and incidence translational energy. We observe the behavior that is far from statistical. Specifically, we find that incidence translation energy has a markedly stronger influence on trapping probability than does molecular vibration for $\mathrm{NO}$ on $\mathrm{Au}\left(\begin{array}{lll}1 & 1 & 1\end{array}\right)$. These results, which to our knowledge are the first of their kind, suggest the value of detailed comparisons to theory. 


\section{Acknowledgment}

This work was supported by a grant from the Department of Energy Office of Basic Energy Sciences, Grant No. DE-FG02-03ER15441.

\section{References}

[1] J.A. Barker, D.J. Auerbach, Surf. Sci. Rep. 4 (1984) 1.

[2] M. Grunze, H.J. Kreuzer (Eds.), Kinetics of Interface Reactions, Springer-Verlag, Berlin, Heidelberg, New York, 1987.

[3] R.I. Masel, Principles of Adsorption and Reaction on Solid Surfaces, Wiley, New York, 1996.

[4] I. Langmuir, Chem. Rev. 6 (1929) 451

[5] P. Kisliuk, J. Phys. Chem. Solids 3 (1957).

[6] P. Kisliuk, J. Phys. Chem. Solids 5 (1958) 78.

[7] A. Cassuto, D.A. King, Surf. Sci. 102 (1981) 388.

[8] W.H. Weinberg, in: M. Grunze, H.J. Kreuzer (Eds.), Kinetics of Interface Reactions, Springer-Verlag, Berlin, Heidelberg, New York, 1987, p. 94.

[9] J.A. Barker, D.J. Auerbach, Faraday Discuss. (1985) 277.

[10] J.A. Barker, C.T. Rettner, J. Chem. Phys. 97 (1992) 5844.

[11] A.M. Wodtke, Y. Huang, D.J. Auerbach, J. Chem. Phys. 118 (2003) 8033.

[12] J. Misewich, H. Zacharias, M.M.T. Loy, Phys. Rev. Lett. 55 (1985) 1919.

[13] H. Zacharias, M.M.T. Loy, P.A. Roland, Phys. Rev. Lett. 49 (1982) 1790.

[14] J.F. Weaver, A.F. Carlsson, R.J. Madix, Surf. Sci. Rep. 50 (2003) 107.

[15] C.T. Rettner, H.E. Pfnur, D.J. Auerbach, J. Chem. Phys. 84 (1986) 4163

[16] C.T. Rettner, D.J. Auerbach, J. Electron Spectrosc. Relat. Phenom. 45 (1987) 335.
[17] C.T. Rettner, D.J. Auerbach, H.A. Michelsen, Phys. Rev. Lett. 68 (1992) 1164.

[18] R.R. Smith, D.R. Killelea, D.F. DelSesto, A.L. Utz, Science 304 (2004) 992.

[19] C.W. Muhlhausen, L.R. Williams, J.C. Tully, J. Chem. Phys. 83 (1985) 2594.

[20] Y. Huang, A.M. Wodtke, H. Hou, C.T. Rettner, D.J. Auerbach, Phys. Rev. Lett. 84 (2000) 2985.

[21] C.T. Rettner, F. Fabre, J. Kimman, D.J. Auerbach, Phys. Rev. Lett. 55 (1985) 1904.

[22] Y.H. Huang, C.T. Rettner, D.J. Auerbach, A.M. Wodtke, Science 290 (2000) 111.

[23] H. Hou, Y. Huang, S.J. Gulding, C.T. Rettner, D.J. Auerbach, A.M. Wodtke, Science 284 (1999) 1647.

[24] A.M. Wodtke, Y. Huang, D.J. Auerbach, Chem. Phys. Lett 364 (2002) 231

[25] Y.H. Huang, M. Sulkes, Rev. Sci. Instrum. 65 (1994) 3868.

[26] C. Amiot, J. Mol. Spectrosc. 94 (1982) 150.

[27] R. Engleman, R.E. Rouse, H.M. Peek, V.D. Baiamonte, Los Alamos National Laboratory, Report No. LA-4364, 1970.

[28] Collisions with metals at high surface temperature do lead to $7 \%$ vibrational excitation probabilities. This is thought to result from a coupling of the decay of thermally excited electron hole pairs in the metal to vibrational excitation of NO. See H. Hou, C.T. Rettner, D.J. Auerbach, et al., Faraday Discuss. 113 (1999) 181, and references therein.

[29] C.T. Rettner, J. Kimman, F. Fabre, D.J. Auerbach, H. Morawitz, Surf. Sci. 192 (1987) 107.

[30] B.D. Kay, T.D. Raymond, M.E. Coltrin, Phys. Rev. Lett. 59 (1987) 2792.

[31] C.L. Kao, J.F. Weaver, R.J. Madix, Surf. Sci. 505 (2002) 115.

[32] M. Asscher, E. Pollak, G.A. Somorjai, Surf. Sci. 149 (1985) 146.

[33] M. Asscher, W.L. Guthrie, T.H. Lin, G.A. Somorjai, Phys. Rev. Lett. 49 (1982) 76. 